\section{Body Temperature and Ambient Pressure, Fully Saturated}

O. Müller-Plathe

Hamburg, Deutschland

\section{Synonym(e) BTPS}

\section{Englischer Begriff BTPS}

Definition BTPS bedeutet Body Temperature and Ambient Pressure, Fully Saturated. BTPS ist eine internationale Konvention, nach der Messgrößen der Lungenphysiologie und der Blutgasanalytik unter den normalerweise in der Lunge herrschenden Bedingungen gemessen werden.

Beschreibung Die BTPS-Bedingungen sind:

- $\mathrm{t}=37^{\circ} \mathrm{C}(310,16 \mathrm{~K})$

- Aktueller Umgebungsluftdruck P(amb)

- Wasserdampfsättigung $\left(\mathrm{pH}_{2} \mathrm{O}\right)$, bei $37^{\circ} \mathrm{C}$ mit $47 \mathrm{mmHg}$ $(6,25 \mathrm{kPa})$
Konsequenzen dieser Festlegung sind unter anderem, dass die Partialdrücke höhenabhängig gemessen werden können und dass Blutgasanalysatoren eine exakte Temperaturkontrolle $\left(37 \pm 0,1^{\circ} \mathrm{C}\right)$ sowie eine Vorrichtung zur Wasserdampfsättigung der Kalibrationsgase aufweisen müssen.

Eine Umrechnung von Blutgaswerten auf die aktuelle Körpertemperatur ist bei den meisten Blutgasanalysatoren problemlos möglich. Ob sie sinnvoll ist oder eher Verwirrung stiftet, ist unter Experten umstritten, da keine gesicherten Erkenntnisse darüber vorliegen, welche Werte zum Beispiel bei starker Hypothermie physiologisch wünschenswert sind. Zur Umrechnung selbst wird auf das IFCC-Dokument verwiesen.

\section{Literatur}

International Federation of Clinical Chemistry (1995) Approved IFCC recommendation on definitions of quantities and conventions related to blood gases and pH. Eur J Clin Chem Clin Biochem 33:399-404 